\title{
Could Some Ball Lightning Observations be Optical Hallucinations Caused by Epileptic Seizures?
}

\author{
Gerald Cooray ${ }^{1}$ and Vernon Cooray ${ }^{*}, 2$ \\ ${ }^{I}$ Department of Neurophysiology, Karolinska Institute, Sweden \\ ${ }^{2}$ Division for electricity, Department of Engineering Sciences, Uppsala University, Sweden
}

\begin{abstract}
The great difficulty of encompassing all observed features of ball lightning into a single theory makes it highly probable that many observations and experiences which have no connection to ball lightning are also categorized as ball lightning experiences. In this note we compare the eyewitness reports of ball lightning and the symptoms of epileptic seizures of the occipital lobe as described in the medical literature and show that a person experiencing such a seizure for the first time may believe that he has witnessed a ball lightning event. Since many of the ball lightning reports are associated with nearby lightning strikes, the possibility that the rapidly changing magnetic field of a close lightning strike could trigger an epileptic seizure is analyzed. The results show that the time derivative of the magnetic field in the vicinity of an intense lightning flash is strong enough to stimulate neurons in the brain. This strengthens the possibility of inducing seizures in the occipital lobe of a person located in the vicinity of lightning strikes.
\end{abstract}

Keywords: Ball lightning, lightning, return stroke, magnetic field, epilepsy, occipital lobe, hallucinations.

\section{INTRODUCTION}

Ball lightning has been seen and described since antiquity and recorded in many places around the globe. Because ball lightning has not been produced in the laboratory and the authenticity of the available photographs is questionable the properties of ball lightning have to be extracted from eyewitness records. Observed properties of ball lightning have been summarized in several books [1-3], in which it is described as being spherical in shape, although other shapes such as teardrops or ovals have also been reported. In rare occasions, ball lightning shaped like rods has also been observed. The diameter of ball lightning is usually $10-40 \mathrm{~cm}$ but occasionally ball lightning as large as one meter has been reported. The life-time of ball lightning is reported to be about 10 seconds, but occasionally ball lightning with a duration of as long as one minute has been observed. Ball lightning may manifest in different colors such as red, redyellow, yellow, white, green and purple. In some cases, the intensity of ball lightning may increase with time and becomes a dazzling white before it disappears explosively. The structure of the ball lightning may vary from one report to another. Sometimes a solid core surrounded by a translucent envelope is reported, in other cases it is a rotating structure or a structure that emits spark-like phenomena. Most ball lightning phenomena are said to move horizontally; however descriptions including motionlessness, a zig-zag movement or moving from a cloud towards the ground can also be found. The speed of movement is reported to be walking speed, i.e. about $1-2 \mathrm{~m} / \mathrm{s}$, and ball lightning that moves

*Address correspondence to this author at the Division for electricity, Department of Engineering Sciences, Uppsala University, Sweden;

E-mail: Vernon.Cooray@angstrom.uu.se, Vernon.Cooray@hvi.uu.se against the wind has also been reported. Some reports describe sharp or acrid odors and $10 \%$ of the observers report sounds associated with the manifestation. The ball may disappear silently or explosively. However, in many cases in which the ball exploded in the vicinity of different objects, no movement of objects or damage to them has been reported.

Ball lightning is usually observed during thunderstorms but a significant number of sightings have also been reported during fine weather without any connection to thunderstorms or lightning. They are also been reported under stressful natural conditions, such as tornadoes, storms and earthquakes. Most of the ball lightning has been sighted indoors, and in one case it was observed inside a commercial aircraft. Ball lightning has also been reported by sailors under stormy conditions but without any lightning. According to observations, balls of lightning can enter or depart from closed rooms, and pass through solid walls and closed windows without any apparent change in its structure and without causing any damage.

There is a wide variety of theories on ball lightning, but so far none of them is able to explain all of the observed features. The great difficulty of encompassing all observed features of ball lightning into a single theory makes it highly probable that many observations and experiences which have no connection to ball lightning are also categorized as ball lightning experiences. In this note we describe the symptoms of an epileptic seizure of the occipital lobe [4] which may convince a person experiencing this for the first time to believe that he has witnessed a ball lightning event. 


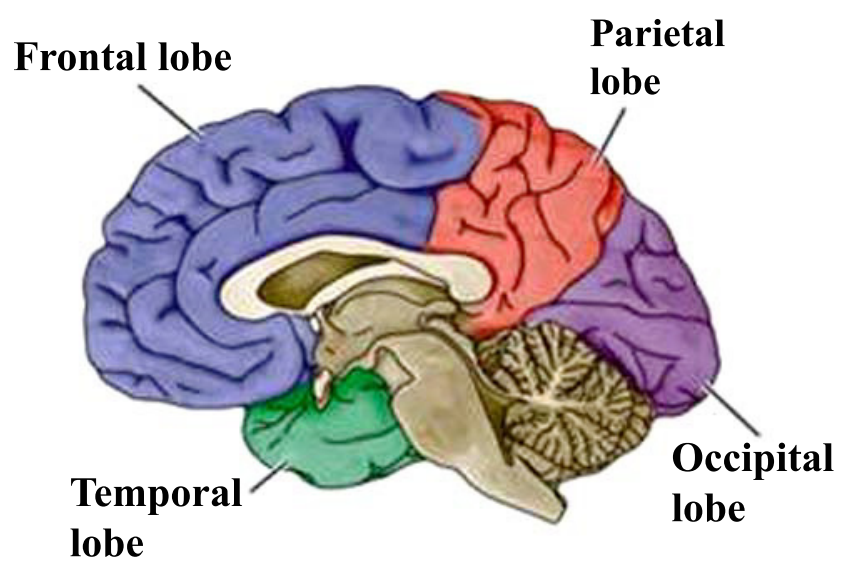

Fig. (1). Different regions of the brain (Adapted from [5]).

\section{EPILEPTIC SEIZURES OF THE OCCI-PITAL LOBE}

An epileptic seizure is an event caused by abnormal, excessive, hyper-synchronous discharges from an aggregate of neurons in the brain. The results of this activity can vary from dramatic convulsive activity to experiential phenomena. Epilepsy describes a condition in which a person has recurrent seizures owing to a chronic, underlying process. Its prevalence has been estimated at 5 to 10 persons per 1000 worldwide [6]. A few percent of all epilepsies have been described as being of occipital origin. Visual hallucinations sometimes occur as a result of a seizure in the occipital, temporo-occipital or temporal lobes of the brain (Fig. 1) $[4,7$, 8]. Elementary visual hallucinations as perceived and drawn by eight patients with occipital epilepsy are shown in Fig. (2). Visual hallucinations in the form of luminous objects, that are either circular or ball like are not uncommon $[4,7]$. In particular luminous balls of different colors, red, yellow, blue and green, moving horizontally from the periphery of the vision to the centre have been described that may appear to be rotating or spinning. Sometimes, the ball may also appear to have a solid structure surrounded by a thin glow or, in other cases, it appears to generate spark like phenomena. When the ball is moving towards the centre of the vision it may increase in intensity and, when it reaches the centre, it can 'explode' illuminating the whole field of vision [4]. During the hallucinations the vision is obscured only in the area occupied by the apparent object. The hallucinations may last for 5 to 30 seconds and, rarely, up to a minute. Occipital seizures may spread into other regions of the brain giving auditory, olfactory and sensory sensations. These sensations can take the form of buzzing sounds, the smell of burning rubber, pain with thermal sensation, especially in the arms and the face, and numbness and a tingling sensation.

In some cases people may experience only one or a few seizures during their lifetime and may not be aware of the reason for the experience. This is specially the case with idiopathic occipital epilepsy, a benign condition affecting predominantly children [9]. This condition can be divided into three subtypes: Early onset, Late onset childhood epilepsy with occipital spikes and Idiopathic photosensitive occipital epilepsy. Children affected with these conditions will often experience only one or a few seizures. Otherwise being in good health, those concerned may categorize their experience as ball lightning encounters. If, as described above, the seizure affected other sensory regions, the resulting experience may appear to have been an electrical effect (the smell of burning, sensation of heat, tingling feeling, etc.) of the ball lightning. Epileptic seizures are a common and important medical problem, with about one in ten persons experiencing at least one seizure at some point in their life. Thus, some of the ball lightning encounters presented in the literature could very well be associated with the experiences of persons who have had an epileptic seizure with visual hallucinations.
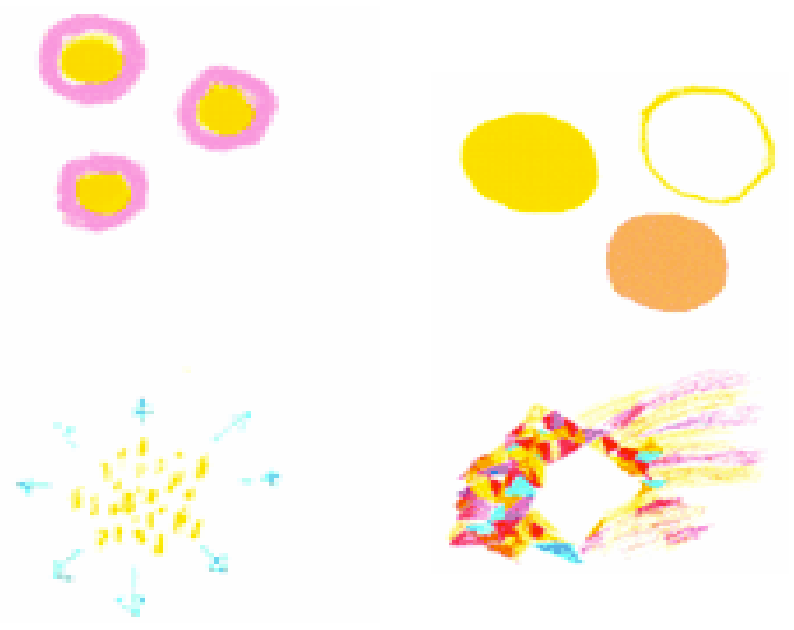

Fig. (2). Elementary visual hallucinations as perceived and drawn by patients with occipital epilepsy [7].

\section{IS THERE A CONNECTION BETWEEN THUNDER- STORMS AND EPI-LEPTIC SEIZURS?}

Many ball lightning sightings have been reported under thunderstorm conditions and on many occasions in the proximity of a lightning strike. Given this fact, a question that would naturally arise is: Could thunderstorms and close lightning flashes trigger an epileptic seizure? It has been reported in the scientific literature that there is an increase in the frequency of epileptic seizures during geomagnetic disturbances, suggesting that exposure to changing magnetic fields could trigger epileptic seizures [10]. More recently, a strong correlation has been observed between the frequency of epileptic seizures and the occurrence of atmospherics in the $10 \mathrm{kHz}$ region [11]. For example, Fig. (3) shows the frequency of epileptic seizures and the rate of occurrence of atmospherics as observed in the study. Since, atmospherics with large amplitudes are generated frequently during thunderstorms, this observation indicates some connection between thunderstorms and epileptic seizures. Even though the exact mechanism of how atmospherics can trigger epileptic seizures is unknown, one may suspect that the magnetic field associated with the atmospheric is the culprit. This is because magnetic fields can penetrate the human body and can generate currents that might interact with the electrical functions of the brain. However, whether the time derivative of the magnetic field of distant atmospherics is strong enough to generate currents that can alter the electrical activity of the brain is a question that needs to be answered. On the other hand, a person standing close to a lightning strike could be 
exposed to a rather high magnetic field time derivative. Indeed, recently, it has been suggested that the time derivative of the magnetic field generated by a close lightning flash may induce heart fibrillations in humans [12]. Today, time varying magnetic fields are being used routinely in medical diagnosis such as Magnetic Resonance Imaging (MRI) and Transcranial Magnetic Stimulation (TMS). The safety precautions necessary in using these techniques have motivated scientists to study the strength of the magnetic field time derivatives necessary for the excitation of nerves and cardiac muscle [13]. Based on these and other observations, scientists have evaluated the threshold levels of magnetic time derivatives necessary for nerve and cardiac stimulation. Let us consider whether the time derivative of the magnetic field in the vicinity of a lightning strike is strong enough to stimulate nerves.

In a lightning flash, the strongest magnetic fields and magnetic field time derivatives are generated by return strokes. Several return stroke models that can be utilized to calculate the magnetic field generated by a return stroke are available in the literature and all these models generate identical results when the point of observation is located in the vicinity of the lightning channel [14]. This is the case since in the vicinity of the channel the magnetic field depends only on the return stroke current flowing at the channel base and it is independent of how the current in the return stroke channel decreases with height. Here we select a simple mathematical model known in the literature as the transmission line model for the calculations [15]. This model requires two inputs, the upward moving speed of the return stroke and the current at the base of the channel. A return stroke speed of $1.5 \times 10^{8} \mathrm{~m} / \mathrm{s}$, which is a typical value, was selected. The current at the channel base of the return stroke was represented by the following waveform which has been adopted in lightning protection standards:

$i=\frac{I}{k} \cdot \frac{\left(t / \tau_{1}\right)^{10}}{1+\left(t / \tau_{1}\right)^{10}} \cdot \exp \left(-t / \tau_{2}\right)$

where $I$ gives the peak current and $\tau_{1}=19.0 \mu \mathrm{s}, \tau_{2}=485 \mu \mathrm{s}$ and $k=0.93$. Calculations were conducted for peak currents of 50, 100 and $200 \mathrm{kA}$. Based on measured lightning current statistics [16], the probability of a negative lightning ground flash maintaining a peak current larger than $50 \mathrm{kA}$ is $20 \%$. The corresponding probability that a current above $100 \mathrm{kA}$ will occur is $1 \%$. Positive ground flashes, which only constitute about $10 \%$ of all ground flashes, have been observed to support currents larger than $200 \mathrm{kA}$. The probability that a given positive ground flash will support a peak current larger than $200 \mathrm{kA}$ is about $20-30 \%$.

The temporal variation of the magnetic field time derivatives of $50 \mathrm{kA}, 100 \mathrm{kA}$ and $200 \mathrm{kA}$ return stroke currents at distances of $2 \mathrm{~m}, 5 \mathrm{~m}$ and $10 \mathrm{~m}$ from the lightning current path are shown in Fig. (4). In general, the striking distance, i.e. the distance of attraction, of lightning flashes supporting these currents is larger than several tens of meters and the chances that lightning flashes of this magnitude will strike ground within $10 \mathrm{~m}$ of a human standing on open ground is rather small. Thus, in the case being considered here, the lightning flash would terminate on the human. However, there are several situations in which a person could be exposed to the magnetic fields generated by strong lightning flashes striking within $10 \mathrm{~m}$. One such example is a person standing within $10 \mathrm{~m}$ of a tree or a high object struck by lightning. A similar scenario could also occur, for example, when lightning strikes a protected building. The current of the lightning flash flows along the down conductors of the lightning protection system and the person could be standing within meters of such a conductor during a lightning strike. Thus, the probability that a human could be exposed to the magnetic field generated by a strong lightning flash within $10 \mathrm{~m}$ is not negligible.

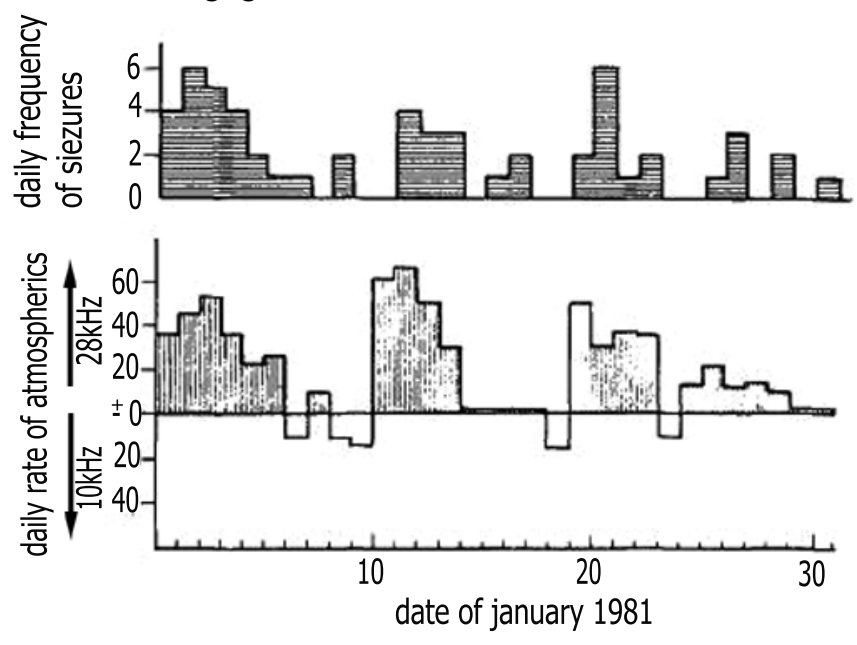

Fig. (3). The correlation between the daily rate of atmospherics and the daily frequency of epileptic seizures [11].

As mentioned previously, scientists have been considering the safety issues related to the medical use of time varying magnetic fields $[13,17]$. From a combination of theory and experiment, they have come up with threshold levels for the magnetic time derivatives necessary for nerve and cardiac stimulation [17]. Fig. (5) shows the rate of change of a magnetic field applied in the form of a ramp required to excite nerves and to induce cardiac stimulation. In this graph the horizontal axis gives the duration of the ramp and the vertical one the rate of change of the magnetic field. The curve for the cardiac stimulation corresponds to the most sensitive population percentile, while the nerve stimulation corresponds to the mean population. Usually, the safe level for magnetic fields in MRI is set to be about three times less than the one corresponding to the nerve stimulation [13]. Note in Fig. (4) that the full width of the magnetic field time derivative is about $20 \mu \mathrm{s}$. The peak values of these magnetic field time derivatives are superimposed in Fig. (5) at $20 \mu \mathrm{s}$ pulse width. First note that the magnetic field derivative of a strong lightning flash striking close to a person could not induce cardiac stimulation. This is in agreement with the conclusions made by Andrew's et al. [18]. On the other hand, observe that, depending on the distance to the current path, the peak values of magnetic time derivatives exceed the values required for nerve stimulation. Recall, too, that the curve for nerve stimulation corresponds to the mean for the 


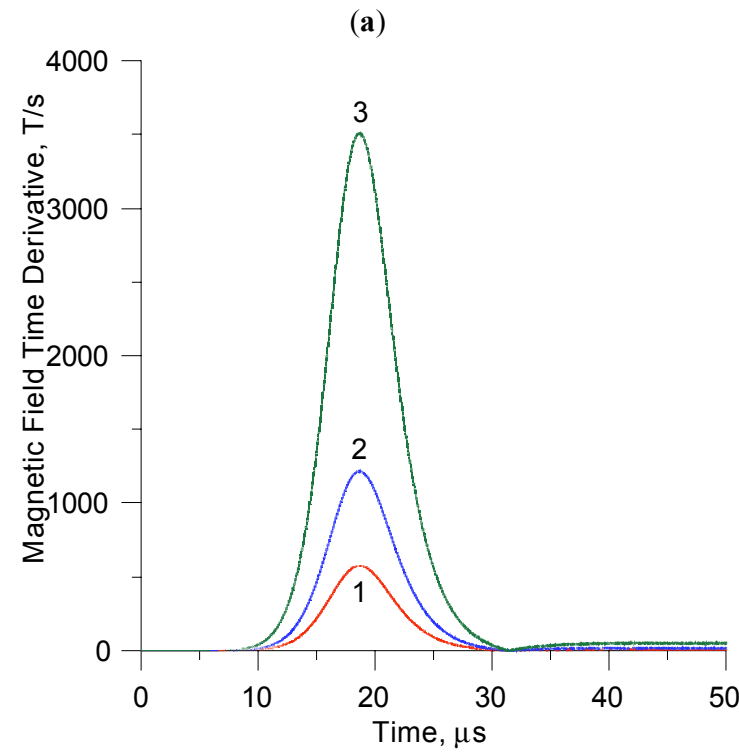

(b)

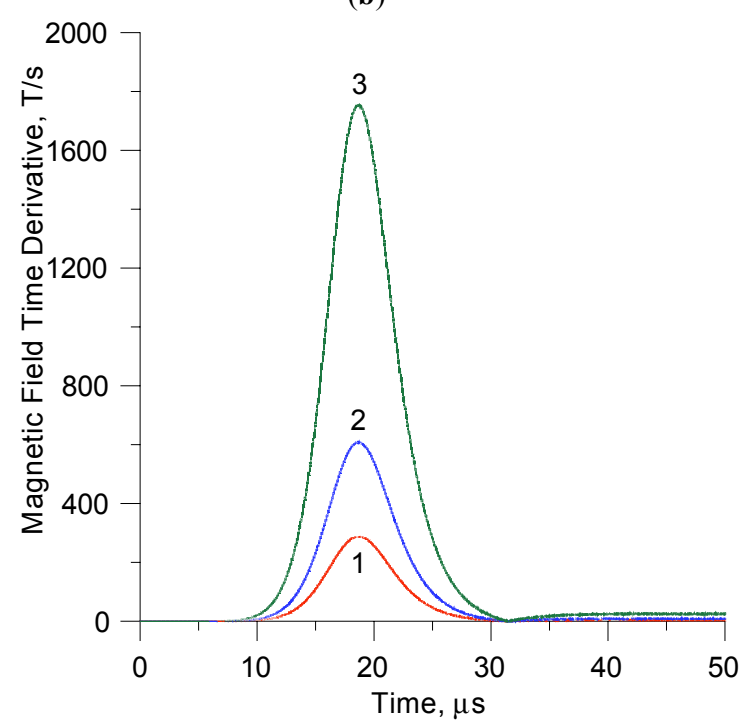

(c)

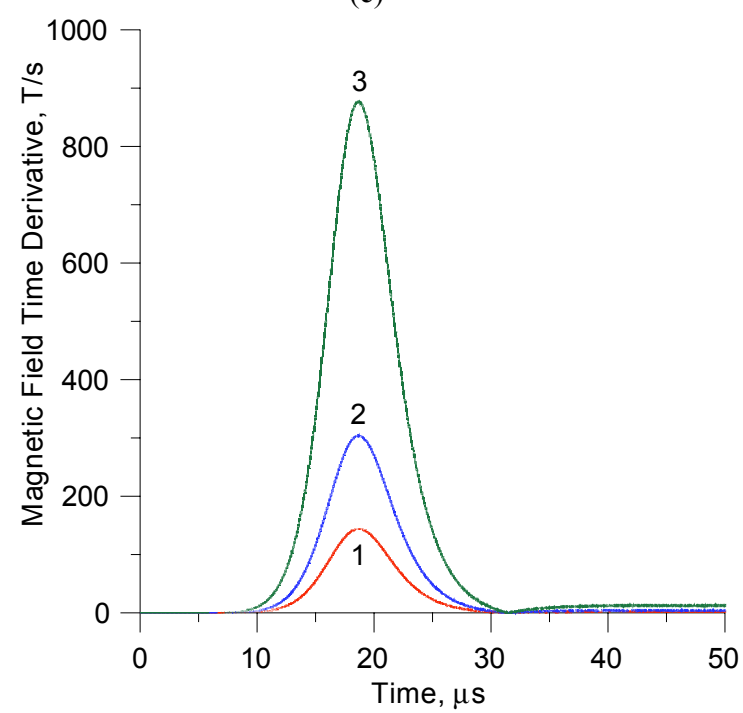

Fig. (4). Magnetic field time derivative at a distance of (a) $2 \mathrm{~m}$, (b) $5 \mathrm{~m}$ and (c) $10 \mathrm{~m}$ from a lightning channel carrying a peak current of $50 \mathrm{kA}$ (curve 1), $100 \mathrm{kA}$ (curve 2) and $200 \mathrm{kA}$ (curve 3).

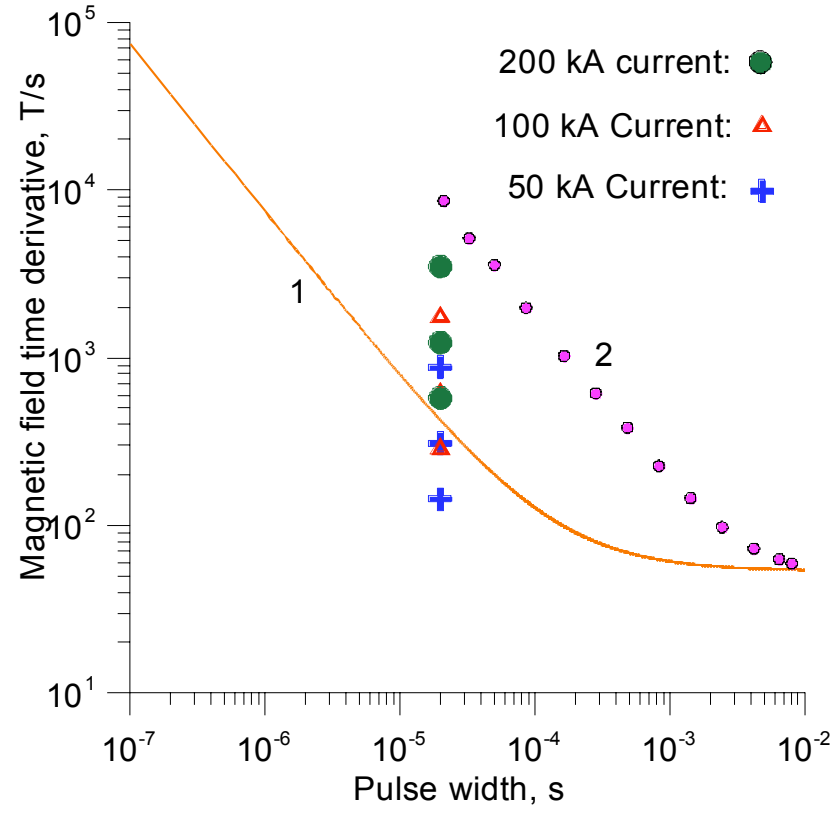

Fig. (5). The rate of change of magnetic field applied in the form of a ramp required to excite the nerves (curve 1) and induce cardiac stimulation (curve 2) [13, 17]. On the same figure, the peak magnetic field derivative for lightning flashes 10,5 and $2 \mathrm{~m}$ from the channel is also depicted for currents of $50 \mathrm{kA}, 100 \mathrm{kA}$ and $200 \mathrm{kA}$.

population, whilst the most sensitive percentile may lie about factor two below this $[13,17]$. The results show that a person located within a few meters of the path of a lightning current could be exposed to a magnetic field derivative that is large enough to stimulate neurons in the brain. This, together with the observed fact that intracranial magnetic stimulation, where the brain is exposed to strong magnetic field derivatives, can cause seizures in epileptic patients [19] suggests to us that the probability of a close lightning flash triggering an epileptic seizure of the occipital lobe is non-zero. It is important to mention that in the analysis presented here we have considered only the first return strokes. Since the lightning parameter which is of interest in the present study is the magnetic field time derivative, the effects of severe subsequent return strokes with large current time derivatives on neuron stimulation cannot be neglected.

Whether an external stimulation such as a strong time varying magnetic field can induce a seizure depends on the 'seizure threshold' of the person under consideration. The 'seizer threshold' refers to the conditions necessary for the production of a seizure. People with a high seizure threshold are more resistant to having seizures than people with a low seizure threshold. The lower the seizure threshold, the more likely the brain cells are to fire at a lower level of stimulus, causing a seizure. One in ten people will have a seizure at some point in their lives, but may not have another one. According to the information provided by American Epilepsy Foundation about 300,000 Americans have a first convulsion each year and about $40 \%$ of them are under the age of 18 . On the other hand about $50 \%$ of children outgrow their epilepsy by the time they become adults. For example, in the case of idiopathic occipital epilepsy which affects predominantly children seizure remission usually occurs within 1-2 years [9]. This indicates that the seizure threshold is lower for 
children and adolescents than adults [20]. Interestingly, it appears that a significant number of ball lightning observations are also reported by children and young adolescents. For example, Abrahamson et al. [21] has presented 41 eyewitness records in which the age at which the ball lightning was encountered is given in 26 reports. Out of this nearly $70 \%$ are from youngsters of age 20 years or below, $46 \%$ are from children of age 15 years or below and $25 \%$ from children less than 10 years of age. If this observation is also true for other data bases it provides further support for the suggestions made in this paper.

\section{CONCLUSIONS}

In this paper we have shown the similarity of the hallucinations associated with epileptic seizures of the occipital lobe and observed features of ball lightning. We suggest the possibility that some of the reported ball lightning observations are hallucinations experienced by people having seizure activity in the occipital lobe. We have also indicated that magnetic fields associated with strong lightning flashes are capable of stimulating neurons in the brain and therefore inducing epileptic seizures, indicating a possible association of between the existence of such reports and thunderstorms and lightning flashes. The possibility that some of the ball lightning reports are contaminated by the hallucinations caused by occipital seizures should be taken into account in the future studies of ball lightning. It may help to separate the real physical facts from the hallucinations paving the way for the future progress in the ball lightning research.

\section{REFERENCES}

[1] Singer S. The nature of ball lightning, Plenum Press, New York, 1971.

[2] Barry JD. Ball lightning and bead lightning, Plenum Press, New York, 1980.

[3] Stenhoff M. Ball lightning-an unsolved problem in atmospheric physics, Plenum Press, New York, 1999.
[4] Blom, Tomson ST, Westerberg CE. Epilepsy in Neurology, Edited by Aquilonius SM, Fagius J, Liber, 2000.

[5] Purves D, Augustine GT, Fitzpatrick D, et al. Neuro Science, Sinauer Associates, Inc. 2001.

[6] Harrison TR. (Ed), Principles of Internal medicine, $14^{\text {th }}$ ed., McGraw-Hill, 1998.

[7] Panayiotopoulos CP. Elementary visual hallucinations, blindness, and headache in idiopathic occipital epilepsy: differentiation from migraine. J Neurol Neurosurg Psychiatry 1999; 66: 536-40.

[8] Bien CG, Benninger FD, Urbach H. Localizing value of epileptic visual auras. Brain 2000; 123: 244-53.

[9] Taylor I, Scheffer IE, Berkovic SF. Occipital epilepsies: identification of specific and newly recognized syndromes. Brain 2003; 126: 753-69.

[10] Rajaram M, Mitra S. Correlation between convulsive seizures and geomagnetic activity. Neurosci Lett 1981; 24: 187-91

[11] Ruhenstroth-Bauer G, Baumer H, Kugler J, Spatz R, Sönning W, Filipiak F. Epillepsy and weather: A significant correlation between the onset of epileptic seisures and specific atmospherics-a pilot study. Int J Biometeor 1984; 28: 333-40.

[12] Cherington M, Wachtel W, Yarnell P. Could lightning injury be magnetically induced? Lancet 1998; 351: 1788.

[13] Reilly JP. Peripheral nerve stimulation by induced electric currents : exposure to time-varying magnetic fields. Med Biol Eng Comput 1989; 27: 101-10.

[14] Cooray V. Mathematical modelling of return strokes, In The Lightning Flash, V. Cooray, Ed. The Institution of Electrical Engineers, UK, 2003

[15] Uman MA, McLain DK. Magnetic field of lightning return stroke. J Geophys Res 1969; 74: 6899-910.

[16] Berger K, Anderson RB, Kroninger H. Parameters of lightning flashes. Electra 1975; 80: 223-37.

[17] Schaefer DJ, Bourland JD, Nyenhuis JA. Review of patient safety in time-varying gradient fields. J Magn Reson Imaging 2000; 12 20-29,

[18] Andrews C, Cooper MA, Kotsos T, Kitagawa N, Mackerras D. Magnetic effect of lightning strokes on the human heart. J Light Res 2007; 1: 158-65.

[19] Tassinari CA, Cincotta M, Zaccara G, Michelucci R. Transcranial magnetic stimulation and epilepsy. Clin Neurophysiol 2003;114: 777-98.

[20] Hay WW, Levin MJ, Sondheimer JM, Deterding RR. Current Diagnosis \& Treatment in Pediatrics, McGraw Hill, New York, 2004.

[21] Abrahamson J, Bychkoy AV, Bychkoy VL. Recently reported sightings of ball lightning: observations collected by correspondence and Russian and Ukrainian sightings. Phil Trans R Soc Lond 2002; A360: 11-35. 